\title{
Métodos para estimar radiação solar na região noroeste de Minas Gerais
}

\author{
Methods for estimating solar radiation in the northwest region of Minas Gerais
}

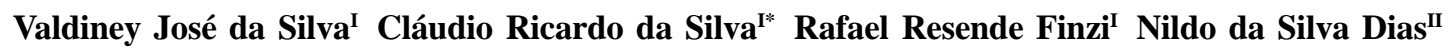

\section{RESUMO}

A radiação solar é um dos elementos responsáveis pela manutenção da vida no planeta. Apesar da sua importância, o registro da radiação solar não é feito na maioria das estações meteorológicas convencionais, uma vez que nem todas dispõem de equipamentos para medi-la, tendo que recorrer a métodos de estimação empíricos. Com o intuito de testar e calibrar equações para estimar a radiação solar, foram coletados dados de quatro estações meteorológicas pertencentes ao Instituto Nacional de Meteorologia (INMET) para representar a região climática noroeste de Minas Gerais, no período de 2008 a 2010. Com base nesses dados, foram testados e calibrados nove modelos para estimar a radiação solar global. Todos os modelos utilizam temperatura máxima e mínima do ar como preditores e dois deles utilizam também a precipitação pluviométrica. Os modelos mais precisos foram de De Jong e Stewart, Bristow e Campbell, Donatelli e Campbell e o modelo dois de Hunt, com raiz quadrada do quadrado médio do erro em torno de $3,1 \mathrm{MJ} \mathrm{m}^{-2} \mathrm{~d}^{-1}$ e coeficiente de determinação em torno de 62\%. No entanto, todos esses modelos apresentaram tendência de superestimação para valores de radiação inferiores à média e subestimação para valores superiores. Portanto, há necessidade de propor novos modelos que minimizem essa tendência.

Palavras-chave: calibração de modelos, irradiância, modelos empíricos.

\section{ABSTRACT}

Solar radiation is a component responsible for maintaining the life on the planet. Despite its importance, solar radiation is not recorded in most conventional meteorological station since not all have equipments to measure it, and then, it is usual to use empirical solar radiation estimation methods.
In order to test and calibrate models for estimating solar radiation data were collected from four meteorological stations belonging to the National Institute of Meteorology (INMET) to represent the climatic region northwest of Minas Gerais for the period from 2008 to 2010. Based on these data, nine models to estimate global solar radiation were tested and calibrated. All the models use maximum and minimum air temperature as predictor and two of them also use rainfall. The most accurate models were De Jong and Stewart, Bristow and Campbell, Donatelli and Campbell and model of two Hunt, with root mean square error about $3.1 \mathrm{MJ} \mathrm{m}^{-2} \mathrm{~d}^{-1}$ and determination coefficient around of $62 \%$. However, all these models tended to have an overestimation for values of radiation below the average and an underestimation for values higher than the average. Therefore there is need to propose new models that minimize this trend.

Key words: calibration of models, irradiance, empirical models.

\section{INTRODUÇÃO}

A radiação solar é a principal fonte de energia responsável pelos processos físicos e biológicos que acontecem na Terra, sendo elemento primordial para o processo de fotossíntese e, consequentemente, pela vida de todos os seres vivos no planeta. Além disso, influencia outros processos ecológicos e ambientais relacionados à temperatura do ar e do solo, afetando a transferência de calor por meio da evaporação e transpiração (SPOKAS \& FORCELLA, 2006). Apesar da sua importância, o registro da radiação

Instituto de Ciências Agrárias e Ambientais (ICIAG), Universidade Federal de Uberlândia (UFU), 38400-902, Uberlândia, MG, Brasil. E-mail: claudio@iciag.ufu.br. *Autor para correspondência.

"Universidade Federal Rural do Semi-Árido (UFERSA), Mossoró, RN, Brasil. 
solar não é feito na maioria das estações meteorológicas convencionais, provavelmente, em função do custo de aquisição, manutenção e calibração dos equipamentos que medem a radiação solar (LIU et al., 2009; ROBAA, 2009; WEISS \& HAYS, 2004). Assim, dados de radiação solar não são facilmente disponíveis como os dados de temperatura e precipitação nas estações meteorológicas (HUNT et al., 1998; RIVINGTON et al., 2005).

Tendo em vista a facilidade de obtenção de alguns elementos meteorológicos e buscando uma alternativa viável para solucionar as dificuldades encontradas na mediação da radiação solar, alguns pesquisadores desenvolveram métodos empíricos que utilizam a temperatura do ar para estimar a radiação solar global. Alguns desses modelos, propostos inicialmente por HARGREAVES (1981) e por BRISTOW \& CAMPBELL (1984), foram ao longo do tempo modificados por outros autores na tentativa de melhorar as estimativas.

Todavia, a calibração desses modelos em cada local é essencial, pois as relações empíricas variam espacialmente. Alguns desses modelos têm sido avaliados em diferentes locais e os coeficientes de calibração variaram consideravelmente (ALMOROX et al., 2011; LIU et al., 2009). Segundo ALMOROX (2009), modelos empíricos são ferramentas adequadas para estimar a radiação solar. Para o autor, esses modelos têm a vantagem de usar dados meteorológicos comumente registrados. Com base no exposto, o trabalho teve como objetivo calibrar e avaliar modelos empíricos de estimativa de radiação solar a partir de dados de temperatura do ar para a região noroeste de Minas Gerais.

\section{MATERIAL E MÉTODOS}

Para a realização deste estudo, utilizaram-se dados da temperatura do ar, precipitação e radiação solar no período de 2008 a 2010, obtidos em estações meteorológicas automáticas pertencentes ao Instituto Nacional de Meteorologia (INMET). Os estudos de calibração e avaliação dos modelos foram realizados para a região noroeste de Minas Gerais, que engloba as estações localizadas nos Municípios listados na tabela 1, que contém a posição geográfica, o período de avaliação e a porcentagem de omissões. As porcentagens de omissões foram calculadas dividindose os dados diários perdidos pelo total de dias do período.

Os dados inicialmente reportados em intervalos horários foram sumarizados em valores diários, obtendo-se valores máximos (Tmáx) e mínimos (Tmín) de temperatura, total de radiação solar incidente $\left(R_{s}\right)$ e Precipitação pluviométrica $(P)$ em cada dia. Em seguida, os dados foram submetidos à avaliação de consistência com base nos critérios de eliminação propostos por LIU et al. (2009): a) dados ausentes para qualquer um dos elementos Tmáx, Tmín ou $R_{s}$; b) Tmáx $\leq$ Tmín; c) $\mathrm{R}_{s} / R_{a}>1$. No período avaliado, o primeiro ano foi utilizado para calibrar os coeficientes e o outro para avaliar o desempenho dos modelos. Os anos foram escolhidos de acordo com as menores omissões. Para as cidades de Buritis e Guarda-Mor, não houve registro de precipitação no período avaliado.

A tabela 2 apresenta os modelos testados: ANNANDALE et al. (2002), BRISTOW \& CAMPBELL (1984), CHEN et al.(2004), De JONG \& STEWART (1993), DONATELLI \& CAMPBELL (1998), HARGREAVES (1981), HUNT et al. (1998). No modelo de Hargreaves (Ha), $R_{s}$ relaciona-se de maneira linear com a raiz quadrada da diferença $\left(\Delta T_{1}\right)$ entre a temperatura máxima (Tmáx) e mínima do ar (Tmín). Esse modelo apresenta apenas um coeficiente ( $a$ ). Porém, CHEN et al. (2004) e HUNT et al. (1998) o apresentaram com dois coeficientes $(a, b)$ (Ch-1, Ch-2 e Hu-1). Já ANNANDALE et al. (2002) introduziram uma correção para a altitude (An). De JONG \& STEWART (1993) introduziram o efeito da precipitação na forma multiplicativa (J-S). HUNT et al. (1998) também apresentaram um segundo modelo com a precipitação e temperatura máxima de forma aditiva (Hu-2).

BRISTOW \& CAMPBELL (1984) propuseram uma equação tendo a temperatura do ar como principal variável de entrada, porém estabelecendo $R_{s}$ uma função exponencial de $\Delta T$ (BC). Embora os coeficientes sejam empíricos, $a$ representa a máxima transmissividade esperada para um dia de céu limpo, a qual depende da altitude e da poluição atmosférica local, e os coeficientes $b$ e $c$

Tabela 1 - Localização geográfica das estações meteorológicas do Instituto Nacional de Meteorologia (INMET) utilizadas no estudo.

\begin{tabular}{lllccc}
\hline Ordem & Cidade & Latitude & Longitude & Altitude & Período \\
\hline 1 & Buritis & $-15,52^{\circ}$ & $-46,43^{\circ}$ & $894 \mathrm{~m}$ & 2008 e 2010 \\
2 & Guarda-Mor & $-17,56^{\circ}$ & $-47,19^{\circ}$ & $616 \mathrm{~m}$ & 2009 e 2010 \\
3 & João Pinheiro & $-17,78^{\circ}$ & $-46,11^{\circ}$ & $870 \mathrm{~m}$ & 2008 e 2009 \\
4 & Unaí & $-16,55^{\circ}$ & $-46,88^{\circ}$ & $631 \mathrm{~m}$ & 2008 e 2009 \\
\hline
\end{tabular}


Tabela 2 - Resumo dos modelos avaliados para estimar radiação solar: (An) ANNANDALE et al., (B-C) BRISTOW \& CAMPBELL, (Ch-1 e Ch-2) CHEN, (J-S) De JONG \& STEWART, (D-C) DONATELLI \& CAMPBELL, (Ha) Hargreaves, (Hu-1 e Hu-2) Hunt.

\begin{tabular}{llll}
\hline Modelo & \multicolumn{1}{c}{ Equação } & Coeficientes & \multicolumn{1}{c}{ Fonte } \\
\hline An & $R_{s}=a\left(1+2,710^{-\mathbf{5}} A l t\right) \sqrt{\Delta T_{\mathbf{1}}} R_{a}$ & $a$ & ANNANDALE et al. (2002) \\
B-C & $R_{s}=a\left(\mathbf{1}-\exp \left(-b \Delta T_{\mathbf{2}}^{c}\right)\right) R_{a}$ & $a, b, c$ & BRISTOW \& CAMPBELL (1984) \\
Ch-1 & $R_{s}=\left(a \sqrt{\Delta T_{1}}+b\right) R_{a}$ & $a, b$ & CHEN et al. (2004) \\
Ch-2 & $R_{s}=\left(a \ln \mathbf{\Delta T _ { 1 }}+b\right) R_{a}$ & $a, b$, & CHEN et al. (2004) \\
J-S & $R_{s}=a \Delta T_{\mathbf{1}}^{b}\left(1+c P+d P^{\mathbf{2}}\right) R_{a}$ & $a, b, c, d$ & DE JONG \& STEWART (1993) \\
D-C & $R_{s}=a\left(\mathbf{1}-\exp \left(-b \frac{\Delta T_{2}^{c}}{\Delta T_{m}}\right)\right) R_{a}$ & $a, b, c$ & DONATELLI \& CAMPBELL \\
Ha & $R_{s}=a \sqrt{\Delta T_{1}} R_{a}$ & $a$ & (1998) \\
Hu-1 & $R_{s}=a \sqrt{\Delta T_{\mathbf{1}}} R_{a}+b$ & $a, b$ & HARGREAVES (1981) \\
Hu-2 & $R_{s}=a \sqrt{\Delta T_{\mathbf{1}}} R_{a}+b T_{\max }+c P+d P^{\mathbf{2}}+$ & $a, b, c, d, e$ & HUNT et al. (1998) \\
\hline
\end{tabular}

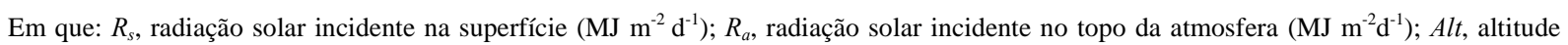
local (m); $\Delta T_{1}$, diferença entre a temperatura máxima e mínima do dia $\left({ }^{\circ} \mathrm{C}\right) ; \Delta T_{2}$, diferença entre a temperatura máxima e a média da mínima dos dois dias consecutivos $\left({ }^{\circ} \mathrm{C}\right) ; \Delta T_{m}$, média mensal de $\Delta T_{2} ; T_{\max }$, temperatura máxima do dia $\left({ }^{\circ} \mathrm{C}\right) ; P$, precipitação (mm); $a, b, c$, $d$ e $e$, coeficientes de calibração dos modelos empíricos (adimensionais).

controlam a taxa na qual $a$ varia com a amplitude térmica. Nesse modelo, a amplitude térmica diária $\left(\Delta \mathrm{T}_{2}\right)$ é obtida com base no valor médio de duas temperaturas mínimas em dias subsequentes, com o objetivo de reduzir o efeito em larga escala da entrada advectiva de massas de ar quente ou fria no local. DONATELLI \& CAMPBELL (1998) propuseram uma correção para o modelo de Bristow e Campbell, para reduzir o efeito da sazonalidade em $\mathrm{R}_{\mathrm{s}}$ (D-C), dividindo $\Delta T_{2}$ pelo $\Delta T$ médio do mês $\left(\Delta T_{m}\right)$.

Os ajustes dos modelos foram realizados pelo método de quadrados mínimos. Neste estudo, de maneira similar aos trabalhos de avaliação de modelos para estimar $R_{s}$ (HUNT et al., 1998; CHEN et al., 2004; WU et al, 2007; ABRAHA \& SAVAGE, 2008; LIU et al., 2009; BORGES et al., 2010), foram utilizadas as seguintes medidas: a) o coeficiente de determinação $\left(R^{2}\right)$ das regressões lineares entre os valores observados e estimados: $R^{2}=\left(\Sigma\left(E_{i}-O_{i}\right)^{2}\right) /\left(\Sigma\left(O_{i}-\bar{O}\right)^{2}\right)$; b) a raiz quadrada do quadrado médio do erro $(R Q M E)$, que pode variar entre 0 ao infinito, sendo que, quanto menor, melhor a estimativa da $R_{s}: R Q M E=\left(\Sigma\left(E_{\mathrm{i}}-O_{\mathrm{i}}\right)^{2}\right) / n$, e; c) o índice de concordância de Wilmott $(d)$, que define a exatidão dos valores estimados em relação aos observados, variando de 0 a 1 , sendo que quanto mais próximo de 1 melhor a estimativa: $d=1-\left(\sum\left(E_{i}-O_{i}\right)^{2}\right) /\left(\Sigma\left(\left|E_{i}-\bar{O}\right|+\left|O_{i}-\bar{O}\right|\right)\right)$; em que $E_{i}$ é a radiação estimada, $O_{i}$ a radiação observada, $\overline{\mathrm{O}}$ a média da radiação observada e $n$ o número de dados.

\section{RESULTADOS E DISCUSSÃO}

A tabela 3 apresenta os coeficientes calibrados dos modelos testados na região noroeste de Minas Gerais. O coeficiente $a$ do modelo An variou entre 0,163 e 0,181 com média de 0,172. No modelo Ha, esse coeficiente variou de 0,166 a 0,186, com média de 0,176 . O modelo B-C apresentou variações entre 0,707 e 0,747; 0,007 e 0,016; e 1,877 e 2,470 para os coeficientes $a, b$ e $c$, respectivamente, com médias de $0,721,0,013$ e 2,099. O coeficiente $a$ desse modelo representa a radiação máxima em um dia claro e seu valor apresentou valores próximos aos observados por MEZA \& VARAS (2000). Essas estimativas são consistentes com valores de coeficientes não calibrados para o mesmo modelo: 0,7 para $a$, o intervalo entre 0,004 e 0,010 para $b$, e 2,4 para $c$ (MEZA \& VARAS, 2000). Para o modelo J-S, os coeficientes $a$ e $b$ variaram de 0,078 a 0,155 e 0,540 a 0,817 , respectivamente. Os coeficientes $c$ e $d$, que mensuram o efeito da precipitação, apresentaram valores muito pequenos, mesmo para as cidades que dispunham de dados de precipitação, mostrando que a precipitação teve pouca influência na estimativa da $R_{s}$, com valores próximos aos obtidos por LIU et al. (2009).

Os coeficientes do modelo Hu-2 variaram consideravelmente entre as cidades, sendo que o coeficiente $d$ teve a maior variação. LIU et al. (2009) encontraram valores ainda mais discrepantes entre os locais avaliados, já que a maior variação foi para o coeficiente $b$. Em geral, os modelos D-C, Ch-1 e Hu-1

Ciência Rural, v.42, n.2, fev, 2012. 
Tabela 3 - Estimativa dos parâmetros dos modelos calibrados para a região noroeste de Minas Gerais: (An) Annandale, (B-C) BRISTOW \& CAMPBELL, (Ch-1 e Ch-2) Chen, (J-S) De JONG \& STEWART, (D-C) DONATELLI \& CAMPBELL, (Ha) Hargreaves e (Hu1 e Hu-2) Hunt .

\begin{tabular}{|c|c|c|c|c|c|c|c|c|}
\hline \multirow{2}{*}{$\begin{array}{l}\text { Modelos } \\
\text { Coeficientes }\end{array}$} & \multirow{2}{*}{$\begin{array}{c}\text { An } \\
a\end{array}$} & \multicolumn{3}{|c|}{ B-C } & \multicolumn{2}{|c|}{ Ch-1 } & \multicolumn{2}{|c|}{ Ch-2 } \\
\hline & & $a$ & $b$ & $c$ & $a$ & $b$ & $a$ & $b$ \\
\hline Buritis & 0,181 & 0,707 & 0,007 & 2,470 & 0,297 & $-0,351$ & 0,421 & $-0,374$ \\
\hline Guarda-Mor & 0,165 & 0,747 & 0,016 & 1,877 & 0,269 & $-0,339$ & 0,401 & $-0,401$ \\
\hline João Pinheiro & 0,179 & 0,708 & 0,014 & 2,139 & 0,288 & $-0,330$ & 0,400 & $-0,335$ \\
\hline Unaí & 0,163 & 0,723 & 0,016 & 1,910 & 0,220 & $-0,193$ & 0,365 & $-0,326$ \\
\hline Média & 0,172 & 0,721 & 0,013 & 2,099 & 0,268 & $-0,303$ & 0,396 & $-0,359$ \\
\hline Modelos & \multicolumn{4}{|c|}{ 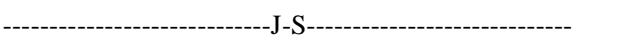 } & \multicolumn{3}{|c|}{------------------D-C------------------ } & $\mathrm{Ha}$ \\
\hline Coeficientes & $a$ & $b$ & $c$ & $d$ & $a$ & $b$ & $c$ & $a$ \\
\hline Buritis & 0,114 & 0,718 & $-0,013$ & $1,6 \mathrm{E}-4$ & 0,693 & 0,022 & 2,999 & 0,186 \\
\hline Guarda-Mor & 0,078 & 0,817 & $-0,013$ & $1,6 \mathrm{E}-4$ & 0,711 & 0,074 & 2,275 & 0,167 \\
\hline João Pinheiro & 0,117 & 0,701 & $-0,008$ & $6,6 \mathrm{E}-5$ & 0,691 & 0,047 & 2,632 & 0,183 \\
\hline Unaí & 0,155 & 0,540 & $-0,014$ & $2,0 \mathrm{E}-4$ & 0,726 & 0,062 & 2,340 & 0,166 \\
\hline Média & 0,116 & 0,694 & $-0,012$ & $1,5 \mathrm{E}-4$ & 0,705 & 0,051 & 2,561 & 0,175 \\
\hline Modelos & \multicolumn{4}{|c|}{----------Hu-1---------- } & - & 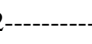 & 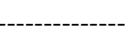 & --------- \\
\hline Coeficientes & $a$ & $b$ & $a$ & $b$ & & & $d$ & $e$ \\
\hline Buritis & 0,170 & 1,774 & 0,120 & 0,375 & & & 0,003 & $-2,591$ \\
\hline Guarda-Mor & 0,182 & $-1,777$ & 0,154 & 0,299 & & & 0,003 & $-6,789$ \\
\hline João Pinheiro & 0,161 & 2,499 & 0,099 & 0,610 & & & 0,002 & $-7,229$ \\
\hline Unaí & 0,181 & $-1,905$ & 0,171 & 0,006 & & & 0,005 & $-0,020$ \\
\hline Média & 0,173 & 0,147 & 0,136 & 0,322 & & & 0,003 & $-4,157$ \\
\hline
\end{tabular}

apresentaram grande variação nos valores dos coeficientes entre os locais, semelhante aos resultados encontrados por LIU et al. (2009). Conforme MEZA \& VARAS (2000), os resultados da calibração mostram que os coeficientes propostos pela literatura não estimam corretamente a média histórica em cada local. Além disso, ajustar ou calibrar os coeficientes dos modelos empíricos para cada cidade em específico, conforme WEISS \& HAYS (2004), implica encontrar um coeficiente específico que melhor se ajusta às condições ambientais do local, que, por sua vez, reduz o erro médio das diferenças entre valores estimados e medidos da radiação solar.

Pode-se observar, na figura 1 , que os melhores resultados para o coeficiente de determinação foram obtidos pelos modelos D-C, J-S, B-C, Hu-2, Ch-1 e Ch-2, com $R^{2}$ em torno de $60 \%$. Assim, pode-se afirmar que esses modelos apresentam uma eficiência em torno de $60 \%$ na estimativa da radiação solar, valores esses abaixo aos encontrados por LIU et al. (2009) e por ABRAHA \& SAVAGE (2008). Porém, os modelos J-S e Hu-2, além de dados de temperatura, usam também os de precipitação, o que implica uma dificuldade a mais no uso desses modelos em comparação aos demais, pois necessitam de equipamentos que registrem também os dados de precipitação.
A pequena diferença entre os modelos de B-C e D-C indica que a modificação proposta ao modelo original não foi muito eficaz. Esses resultados concordam com os obtidos por LIU et al. (2009) ao verificarem que a modificação do modelo apresentou pouca ou nenhuma melhora em relação ao modelo original. Além disso, foi observado que todos os modelos tendem em superestimar os menores valores da radiação solar global até a média $\left(18 \mathrm{MJ} \mathrm{m}^{-2} \mathrm{~d}^{-1}\right)$, e a subestimar os valores acima desta. Isto se deve provavelmente à concentração dos dados em torno deste valor ao longo do ano, que favoreceu a baixa presença de valores extremos, principalmente abaixo deste valor.

Apesar de ser uma adaptação do modelo Ha, o modelo de An foi o que apresentou o resultado menos favorável, com $R^{2}$ de 0,50 , seguido pelos modelos Hu-1 e o Ha. Já o modelo de Ch-1 apresentou desempenho melhor que o Ha, $\operatorname{com} R^{2}$ de 0,58 . Conforme estudos feitos por HUNT et al. (1998), no Canadá, o modelo Ha apresentou melhores resultados para $R^{2} \mathrm{e}$ $R Q M E$ em relação ao modelo de B-C, diferentemente dos resultados obtidos nesse estudo. Também ALMOROX (2009) obteve bom desempenho para o modelo Ha. O autor afirmou que esse modelo pode ser usado com acurácia para estimar a radiação solar 


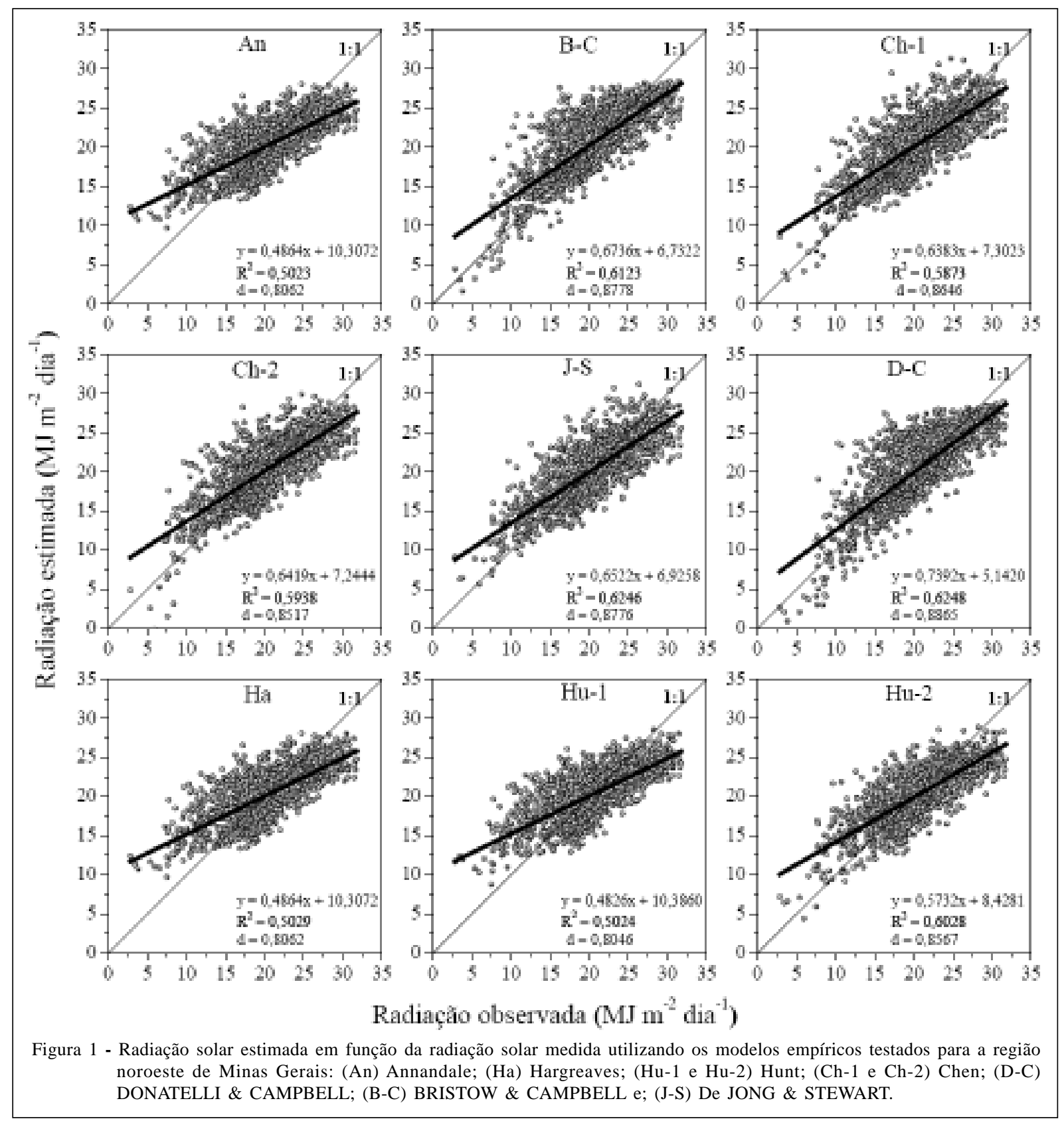

quando se dispõe somente de dados de temperatura e quando os modelos não podem ser calibrados para as condições climáticas locais.

Os resultados do índice de concordância $d$, também expressos na figura 1, confirmam a superioridade dos modelos D-C, B-C, J-S, Ch-1, Hu-2 e Ch-2, com valores acima de 0,85 . Isso indica que a radiação solar global estimada por esses modelos apresenta concordância acima de 85\% com os medidos. Os modelos de Ha, An e Hu-1 concordam em 80\% com os medidos, indicando uma superioridade de $5 \%$ daqueles modelos em relação a esses. Os valores de $d$ obtidos estão entre os encontrados por BORGES et al. (2010) em Cruz das Almas, BA, com o modelo Ha não calibrado.

Os resultados de $R M Q E$ ilustrados na figura 2 demonstram que os modelos J-S, B-C, D-C, Hu-2, Ch1 e Ch-2, novamente, foram os melhores em relação à concordância entre valores observados e preditos. Esses valores de $R Q M E$, em geral, foram inferiores aos obtidos por LIU et al. (2009), porém próximos aos comumente encontrados na literatura, mesmo para os 


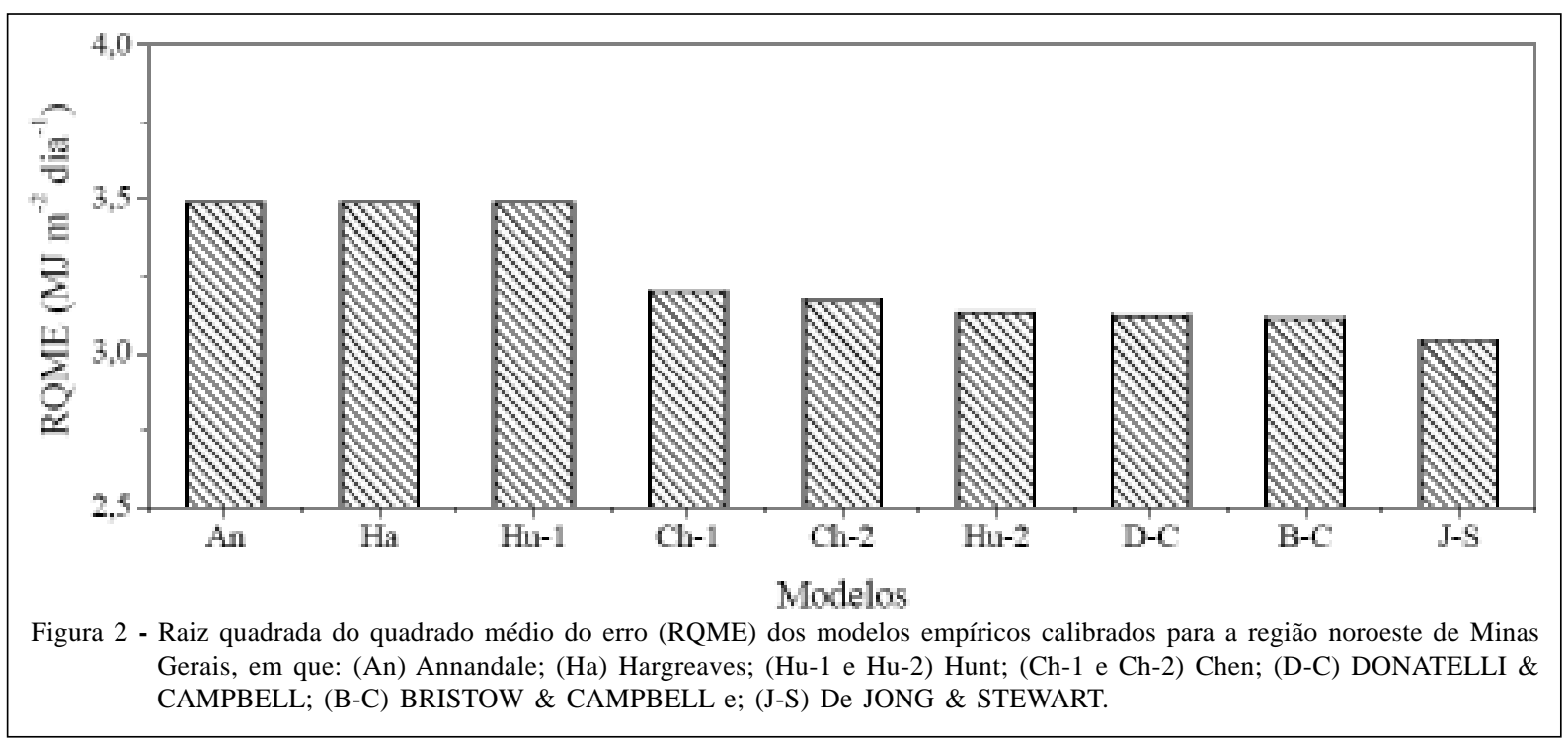

modelos com maiores RQME (CHEN et al., 2004; ABRAHA \& SAVAGE, 2008) e, considerando que os dados de temperatura são mais fácies de serem obtidos, para os estudos que não requerem grande exatidão na estimativa da radiação solar incidente na superfície, tais modelos tornam-se atrativos, principalmente pela simplicidade de cálculo.

Um fator a considerar é que os modelos avaliados foram calibrados com dados de temperatura máxima e mínima do ar de apenas um ano e isso pode influenciar no desempenho desses modelos em caso de ocorrência de um ano atípico, quer seja nas suas calibrações ou avaliações. No entanto, alguns desses modelos, como o de Bristow e Campbell, foram propostos com calibração de seus coeficientes com apenas um ano de estudo. Ademais, os valores dos coeficientes aqui encontrados estão próximos aos encontrados em literaturas com desempenhos também semelhantes quando das avaliações.

\section{CONCLUSÃO}

Os modelos J-S, B-C, D-C, Hu-2, Ch-2 e Ch1 são os mais precisos para estimar a radiação solar diária para a região noroeste de Minas Gerais. O modelo Ha, não calibrado, em função da sua simplicidade, pode ser usado para obter estimativas diárias da radiação solar quando não se requer grande precisão. Os modelos avaliados superestimam a radiação numa faixa entre valores mínimos e médios para a região considerada e subestimam os referidos valores acima desta. Há necessidade de proposição de modelos mais adequados onde não ocorram os padrões dessa tendência, observados em todos os modelos avaliados.

\section{REFERÊNCIAS}

ABRAHA, M.G., SAVAGE, M.J. Comparison of estimates of daily solar radiation from air temperature range for application in crop simulations. Agricultural and Forest Meteorology, v.148, p.401-416, 2008. Disponível em: <http:// www.sciencedirect.com/ science/article/pii/ S0168192307002626>. Acesso em: 20 jun. 2011. doi: 10.1016/ j.agrformet.2007.10.001.

ALMOROX, J. et al. Models for obtaining daily global solar radiation with measured air temperature data in Madrid (Spain). Applied Energy, v.88, p.1703-1709, 2011. Disponível em: <http://www.sciencedirect.com/science/article/pii/ S0306261910004666>. Acesso em: 10 jun. 2011. doi:10.1016/ j.apenergy.2010.11.003.

ALMOROX, J. Estimating global solar radiation from common meteorological data in Aranjuez, Spain. Tübitak, v.35, p.5364, 2009. Disponível em: <http://journals.tubitak.gov.tr/ physics/issues/fiz-11-35-1/fiz-35-1-7-0912-20.pdf $>$. Acesso em: 05 abr. 2011. doi: 10.3906/fiz-0912-20.

ANNANDALE, J.G. et al. Software for missing data error analysis of Penman-Monteith reference evapotranspiration. Irrigation Science, v.21, p.57-67, 2002.

BORGES, V.P. et al. Avaliação de modelos de estimativa da radiação solar incidente em Cruz das Almas, Bahia. Revista Brasileira de Engenharia Agrícola e Ambiental, v.14, n.1, p.74-80, 2010. Disponível em: <http://www.scielo.br/pdf/ rbeaa/v14n1/v14n01a10.pdf>. Acesso em: 10 jun. 2011. doi: $10.1590 /$ S1415-43662010000100010.

BRISTOW, K.L.; CAMPBELL, G.S. On the relationship between incoming solar radiation and daily maximum and minimum temperature. Agricultural and Forest Meteorology, v.31, p.159-166, 1984. Disponível em: <http:// w w w. sciencedirect.com/science/article/pi i / 0168192384900170>. Acesso em: 06 mar. 2011. doi: 10.1016/ 0168-1923(84)90017-0. 
CHEN, R.S. et al. Validation of five global radiation models with measured daily data in China. Energy Conversion and Management, v.45, n.1759-1769, 2004. Disponível em: $<$ http://www.sciencedirect.com/science/article/pii/ S019689040300270X>. Acesso em: 06 mar. 2011. doi: 10.1016/j.enconman.2003.09.019.

DE JONG, R.; STEWART, D.W. Estimating global solar radiation from common meteorological observations in western Canada. Canadian. Journal. Plant Science, v.73, p.509518, 1993.

DONATELLI, M.; CAMPBELL, G.S. A simple model to estimate global solar radiation. In: FIFTH CONGRESS OF THE EUROPEAN SOCIETY FOR AGRONOMY, 1998, Nitra, Slovakia Republic. Proceeding... Nitra: Fifth Congress of the European Society for Agronomy, 1998. v. 2, p.133-134.

HARGREAVES, G.H. Responding to tropical climates. In: FOOD AND CLIMATE REVIEW, THE FOOD AND CLIMATE FORUM, Aspen Institute for Humanistic Studies, 1980-1981, Boulder, Colo. Proceedings... Boulder: Aspen Institute for Humanistic Studies, 1981. p. 29-32.

HUNT, L.A. et al. Estimation of solar radiation for use in crop modeling. Agricultural and Forest Meteorology, v.91, n.34, p.293-300, 1998. Disponível em: <http:// w ww.sciencedirect.com/s cience/article/pi i/ S0168192398000550>. Acesso em: 15 maio, 2011. doi: 10.1016/S0168-1923(98)00055-0.

LIU, X. et al. Calibration of the Angstrom-Prescott coefficients (a, b) under different time scales and their impacts in estimating global solar radiation in the Yellow River basin. Agricultural and Forest Meteorology, v.149, p.697-710, 2009. Disponível em: <http://www.sciencedirect.com/science/article/pii/
S0168192308003031>. Acesso em: 06 mar. 2011. doi: 10.1016/j.agrformet.2008.10.027.

MEZA, F.; VARAS, E. Estimation of mean monthly solar global radiation as a function of temperature. Agricultural and Forest Meteorology, v.100, p.231-241, 2000.

RIVINGTON, M. et al. Evaluation of three model estimations of solar radiation at 24 UK stations. Agricultural and Forest Meteorology, v.132, p.228-243, 2005. Disponível em: <http:/ / w w w.sciencedirect.com/s cience/article/pi i / S0168192305001577>. Acesso em: 10 jun. 2011. doi: 10.1016/ j.agrformet.2005.07.013.

ROBAA, S.M. Validation of the existing models for estimating global solar radiation over Egypt. Energy Conversion and Management, v.50, p.184-193, 2009. Disponível em: <http:/ / w w w.sciencedirect.com/science/article/pi / S019689040800280X $>$. Acesso em: 10 jun. 2011. doi: 10.1016/j.enconman.2008.07.005.

SPOKAS, K.; FORCELLA, F. Estimating hourly incoming solar radiation from limited meteorological data. Weed Science, v.54, n.1, p.182-189, 2006.

WEISS, A. HAYS, C.J. Simulation of daily solar irradiance. Agricultural and Forest meteorology, v.123, p.187-199, 2004. Disponível em: <http://www.sciencedirect.com/science/ article/pii/S0168192303002946>. Acesso em: 10 jun. 2011. doi: 10.1016/j.agrformet.2003.12.002.

WU, G. et al. Methods and strategy for modeling daily global solar radiation with measured meteorological data - A case study in Nanchang station, China. Energy Conversion and Management, v.48, p.2447-2452, 2007. Disponível em: <http://www.sciencedirect.com/2 science/article/pii/ S0196890407001100>. Acesso em: 10 jun. 2011. doi: 10.1016/ j.enconman.2007.04.011. 